\title{
Naturalism: The Epistemological Characteristics of Laozi's Management Philosophy
}

\author{
Qiu Daidong 1,*

\begin{abstract}
${ }^{1}$ Basic Teaching Department of Guangzhou College of Technology and Business, Huadu, Guangzhou, Guangdong, China *qudaidong@163.com
\end{abstract}

\begin{abstract}
The epistemology of Laozi's management philosophy transcends the dichotomy of qualitative and quantitative. It puts the management object out of the time coordinate set by man, and regards the subject and object of management as the natural thing of "Tao" and also makes the subject and object of management subject to the natural law. The epistemology of Laozi's management philosophy has distinct naturalistic characteristics. The naturalistic characteristics of the epistemology of Laozi's management philosophy, to a certain extent, make up for the deficiency of the empirical logic that attaches importance to linear causality. Its profound connotation provides new ideas for the reform and innovation of management theory.
\end{abstract}

Keywords: Laozi, management philosophy, epistemology, naturalism

\section{自然主义:《老子》管理哲学的认识论特征}

\author{
邱代东 ${ }^{1, a}$
}

${ }^{1}$ 广州工商学院基础教学部, 花都, 广州, 广东, 中国

a qiudaidong@163.com

\section{摘要}

《老子》管理哲学的认识论跨越了人为的定性与定量二分法, 将管理对象放在人为设置的时间坐标之 外，将管理主客体同视为 “道” 化的自然之物并受制于自然规律，具有鲜明的自然主义特征。《老子》 管理哲学认识论的自然主义特征, 在一定程度上弥补了重视线性因果关系的实证逻辑的不足, 其内涵 的深沉为管理理论的变革和创新提供了新的思路。

关键词: 《老子》, 管理哲学, 认识论, 自然主义

\section{1. 前言}

认识论 (epistemology) 即个体的知识观, 也即 个体对知识和知识获得所持有的信念, 主要包括有关 知识结构和知识本质的信念和有关知识来源和知识判 断的信念, 以及这些信念在个体知识建构和知识获得 过程的调节和影响作用, 长久以来一直是哲学研究的 核心问题。 ${ }^{[1]}$ 在管理学领域里, 认识论指的是人类对 管理的认识来源、认识能力、认识形式、认识过程和 认识真理性问题的理论。《老子》的管理认识论将事物 与现象放置在定性与定量之外, 从个体的分析过渡到 整体的认识上, 以超越时间维度的方式分析管理规律。

\section{2. 定性与定量之外}

管理学的研究, 通常会有定性研究与定量研究之 分。定性研究用文字语言进行研究的描述, 常常用于
对管理现象进行高度抽象的描述时使用，用来揭示的 对管理事物规律性的认识。定量则是用数学语言进行 研究的表述, 常运用数学、统计学、计量学等科学方 法对管理中出现的现象进行量化分析, 在量化分析的 基础上得出对客观事物的规律性认识。《老子》在对管 理现象的认识过程中, 往往兼用两者, 并不明确区分 定量研究与定性研究, 偶尔还将两者结合进行分析研 究。因此, 《老子》管理哲学的认识论带有一定的 “混 沌” 色彩, 把管理现象的探索放置在了定性研究与定 量研究二分法的范畴之外。

《老子》在提出运作自然万物的机制的时候，从 量的层面上对自然万物的性质进行了定性的指认。《老 子》第三十四章说: “万物恃之以生而不辞, 功成而不 名有。衣养万物而不为主, 常无欲可名于小。”其中的 万物并非一万种事物, 而是指存在于自然中所有的事 物。“大道泛兮，其可左右” (第三十四章), “这是从 总体上说道体无所不在、无所不有的普遍性。” ${ }^{[2]}$ 实际 上, 这里的 “泛” 可以认定为 “全部”, 或者说所有。 因为, 《老子》认为天下所有之物, 都是法自于道, 也 
都是在道的管辖之下, 是道衍生出所有的事物。“大道 泛兮” 对事物数量上的圈定是模糊的, 从模糊的量上 为道作为机制发挥作用进行了性的认定，即事物都效 法于道, 道存在于所有的事物之中。

《老子》第四十章说: “天下万物生于有, 有生于 无。” 有与无, 是两个相对的概念, 自然万物是从有 生发出来的, 而有则来源于无。这里的无, 不是虚无, 而是最初的生命的机制。老子用溯源的方式为自然万 物找到源头, 是为了说明, 自然万物并无本质上的区 别。所以，万物与一、二、三并无本质上的不同，都 同取自于道，而道则效法自自然。这种看待世界的方 式, 是以一比拟万物, 挖掘事物本身的规律, 而这个 规律也就是自然之道。因此, 在进行管理活动的过程 中, 也不应该区别对待不同的事物, 要正面它们内在 一样的本质, 也要意识到统辖万物的道的存在。《老子》 在考察自然万物与有和无的关系时, 超越了简单的质 变与量变的思路及常用的分类思想。《老子》对管理本 体形而上的认识, 与逻各斯中心主义探源的方式相同。 对自然万物趋于本源的探索, 以实证主义科学实验的 方式是不可行的。巴斯卡认为, 科学实在论 “依循常 见的、物理主义式的科学方法来进行科学研究, 所得 到的科学成果 (特别是科学理论及其经验观察证据) 都只是认知成果。既然如此, 它们只在认识论层面有 效。” ${ }^{\left[{ }^{3}\right]}$ 老子在思考问题时, 并不以定性作为依据, 也 不以定量作为依据, 同时也没有进行量变到质变的探 索, 他的方法迈出了定性与定量研究二分法以外。

要客观正确的看待事物, 需要超越量化的思维, 事物本质上的相同并不需要人们从数量上去佐证。《老 子》第四十七章说: “不出户知天下。不窥牑见天道。” 《老子》认为, 了解自然的规律并不需要观察或认识 大量的事物, 换言之, 观察世界和认识世界不能完全 用归纳法, 不能单纯从量上面去进行统计分类, 而是 要进行适度的提炼, 结合演绎形成有效的认识。

自然界的事物及其现象往往很难以物理单位进行 量化的衡量, 而是多以是形容词或副词表达程度。《老 子》注意到了这一点, 在观察自然规律指导人类社会 管理实践的过程中, 采用的是有程度之分的词, 而非 在数字上进行自然规律的判定。《老子》第二十三章说: “希言自然。故飘风不终朝, 骤雨不终日。孰为此者? 天地。天地尚不能久, 而况于人乎? ” 希、终、久等 都是表示程度的词, 用以表述自然事物变化的。第十 三章内容上想要说明, 天地都不能保持某种状态的恒 久不变, 何况人类。因此, 人们在管理实践中以自然 为参照, 减少过多的管理行为, 以自然的现象作为克 制自我的定性要求。

《老子》在提出以生存为前提和最终目标的管理 目的时候, 用数字比例对过于追求长寿同样会招致死 亡的道理进行了说明。《老子》第五十章说: “生之徒, 十有三。死之徒, 十有三。” 意思是人类, 活到正常寿 命的有十分之三; 非正常死亡的有十分之三; 还有十 分之三的人是为了追求享受和长寿而采取各种活动而 导致自身死亡的。十分之三的比例并不精确, 甚至还 不准确, 却大致表达了彼时人们生存死亡的实际状况。
老子借用这个比例, 说明过分地追求享受和长寿, 其 实是违背自然之举, 是人为凌驾在了自然之上, 也就 得不到自然的好结果, 看似从数量上下了结论, 其实 是采用了定性的方式。《老子》管理哲学并不一味的追 求定性与定量, 而是将两者相结合以便更为准确的观 察在道的统辖下的自然世界, 以得出如何管理好自身 的原则和逻辑。

\section{3. 共时与历时之外}

《老子》中不乏古今之说, 但在观察外在事物和 人类社会时, 它往往会摒弃时间因素, 假定结论不在 特定历史时间和社会环境之中。《老子》用溯源的方式, 观察并寻找自然万物的起源: “万物并作, 吾以观复。 夫物芸芸各复归其根” (第十六章)。在观察中帮助万 物返回其根, 也就是回到原初的时间, 即迈向时间轴 的左端。同时, 《老子》又认为回到根就是回到了 “道”, 就能掌握亘古不变的规律和真理, 那也就能处理好现 在, 也能很好的面向未来, 最终实现 “没身不殆”。对 于管理而言, 管理者掌握了道, 也就能回溯到过去, 懂得道作为机制的作用原理, 进而理清现在, 也能很 好地处理未来。

《老子》对于真理的追求, 让它自身在对管理的 认识中出现了将历时变为共时的行为, 即管理并不随 时间而有所改变。《老子》认为管理应该像道一样穿越 时间和社会变迁，管理中存在一个长久不变的、还能 迈过实践积累的机制。因此，《老子》在描述管理之道 的时候，赋予了管理更多形而上的特征。《老子》第二 十一章: “孔德之容惟道是从。道之为物惟恍惟惚。惚 兮恍兮, 其中有象。恍兮惚兮其中有物。窈兮冥兮, 其中有精。其精甚真。其中有信。自古及今, 其名不 去以阅众甫。吾何以知众甫之状哉! 以此。” 这章在描 述道的时候, 将古与今放置在了同一个层面, 谈道作 为机制具备的外形特征及作用特色。它认为, 要观察 事物的起源, 从古至今都离不开道, 道乃恒久不变的, 作为机制发挥的作用也可以跨过时间的影响, 成为经 得起时间考验的真理。

在论述管理原则的时候, 《老子》往往将古今现象 一并分析总结。《老子》第六十五章说: “古之善为道 者, 非以明民, 将以愚之。民之难治, 以其智多。故 以智治国, 国之贼。不以智治国, 国之福。知此两者, 亦稽式。常知稽式, 是谓玄德。玄德深矣、远矣! 与 物反矣。然后乃至大顺。” 《老子》以古之经验来指导 当下的国家治理, 提出古时的治理者是如何做的、可 以怎样地用来指导今天的国家管理实践。古时善于治 理国家的得道者常常让民众保持淳朴，而不是狡诈; 民众有过多的智谋, 会影响国家的治理, 不听从治理 者的安排和告诫, 由此, 《老子》认为今人同样应该摒 弃对民众智慧的过度启发。民众过多的智谋, 是民众 摆脱蒙昧的标志, 而万事万物的根源自于道, 道不主 张有过多的人为干预; 如若民众智谋过多，便会产生 过多的人为因素，进而影响国家治理者管理政策的实 
施。

《老子》认识的管理，是跨越时间的管理，不受 社会变迁和时代改变影响的管理。《老子》认为管理者 的能力不在于从现实中吸取经验教训, 也不在于如何 解决当下新出现的管理难题与管理困境。它认为要真 正解决现实管理中出现的问题，是不能只看现在的, 而是要依据现实中的情况去溯源, 找到现象的本源, 并将过去与现在并置在一起进行观察和处理。因此, 《老子》才会在第二十八章中提出: “为天下式, 常德 不忒, 复归于无极”, 即天下之楷模, 是能够长久存在, 也能够回归于最初的道。管理者守住了道, 也就能够 在管理活动中始终以最初的、自然的方式进行管理, 从而保证管理成效能够不跨越自然的范畴, 而得到长 久的保持。

《老子》认识管理现象时对时间作为社会发展重 要元素的刻意忽视, 很大程度上是因为老子溯源的思 维方式。老子在思考问题解决方案的时候, 常常以事 物最初的形态为参照对象。牛贵琥认为, 老子的求源 式思维很值得关注。正因为使用了求源思维, 老子在 看问题的时候就不会停留在问题的表面, 比一般的思 想家看得既深刻又有远见。 ${ }^{[4]}$ 在面对五花八门的现象 时, 如若不会到问题发生的源头, 是没有办法很好的 处理问题。国家治理, 事务繁多, 需要有一以贯之的、 核心的理念, 否则便会出现朝令夕改的现象, 不仅看 上去管理混乱, 实际上管理组织的基础也不稳妥。此 外, 《老子》忽略时代的管理认识观, 还与他以生存为 前提和目标的管理目的有着密切的关系。老子思考问 题习惯回到人类社会和自然界的源初, 是重视生存的 表现, 想要回避冲突和战争。冲突和战争的出现, 和 各个国家发展扩张又有直接的联系。老子不以发展的 眼光看待组织或社会, 抛开时间的影响思考国家的出 路, 是希望人们回到过去的、生活安稳的、没有祸患 的社会状态。也正因如此, 老子提倡 “小国寡民”, 希 望 “民之老死不相往来” (第八十章)。然而, 时代的 发展是不可阻挡的, 站在历时与共时之外考虑国家治 理，是过于理想化，不符合事物发展逻辑的。

\section{4. 从个体到整体}

《老子》管理哲学没有对管理主体与客体进行刻 意的区分, 书中很多管理思想所提出来的要求, 既针 对管理者, 也针对被管理者。《老子》第十三章说: “吾 所以有大患者, 为吾有身, 及吾无身, 吾有何患”, 因 此 “贵以身为天下, 若可寄天下; 爱以身为天下, 若 可托天下。” 看似老子强调 “身” 之重要, “但实则是 将 “贵身” 置于与天下同等重要的地位, 目的在于明 侯王和圣人, 不可因外在的名利得失而迷失本性”。 ${ }^{[5]}$ 抛弃对个体过分的关心和照顾，是为了更好的照顾整 体。作为国家的管理者, 照顾好整体指的就是照顾好 国家, 即国家照顾好了, 作为其中的个体自然也就会 有好的结果。可见, 在《老子》中, 管理行为的两端 管理者与被管理者, 其实就是一个整体, 没有绝
对的主客体之分；管理的认知与格局是站在整体的立 场, 以第三人称的视角进行建构的。实际上, 《老子》 模糊了个体与整体之间的利益界限, 要求管理者能够 做到以整体利益为先, 个人利益为后。在此认识下, 才会有 “既以为人己愈有, 既以与人己愈多” (第八十 一章）的结论，也才会对国家管理者有 “为而不争” 的要求。

《老子》认为管理应以个体作为切入口, 以推己 及人的方式，进而做好整体、全面的管理。“上礼为之 而莫之以应，则攘臂而扔之。故失道而後德。失德而 後仁。失仁而後义。失义而後礼。” (第三十八章) 指 的就是管理者自身是否符合 “道”，进而拥有管理的 “德”。如果管理者不具备在上等的仁、义、礼, 那么 就会产生有意而为之的仁、义、礼。故意为之的礼是 忠信薄弱下的产物, 即 “忠信之薄而乱之首”, 是管理 出现混乱的开始。因此要做好管理工作, 首先要提高 管理者自己的德行, 具体来做就是: “处其厚不居其薄; 处其实，不居其华” (第三十八章)。在推己及人的过 程中，管理者可以通过 “以身观身，以家观家，以乡 观乡, 以邦观邦，以天下观天下” (第五十四章) 来实 现, 也就是由自身来观察认识其他的人, 由自己的家 庭来观察认识其他家庭, 由自己所处的乡里来观察认 识其他的乡里, 还可以由自己的国家来认识其他的国 家, 最终可以实现由一个天下来观察认识另一个天下。 在《老子》看来, 从个体到整体, 是认识事物的一种 普遍规律, 也是管理国家过程中认识国家社会的一种 有效途径。因此, 老子说 “吾何以知天下然哉? 以此” (第五十四章)。

《老子》认为好的管理者应该削弱个人的意志, 不将个人的意志强加于人, 是谓: “圣人无常心。以百 姓心为心。(第四十九章) “圣人治理天下, 应收玫自 我意志, 削减自我欲望。” ${ }^{[6]}$ 国家管理者照顾老百姓的 想法和观念, 其最终目的是为了天下, 也就是为了作 为整体的国家和社会, 因而回避了自己的私欲。也因 为回避了自己的私欲, 管理者才能做到把心沉淀下来, 以无为的方式进行管理并且不生事端, 被管理者也就 能够 “自正”、“自富”和 “自足”, 最终达到 “自化”。

《老子》中对管理的认识, 是以 “道”为出发点 的。管理者管理水平的高低在于他认识事物是否遵循 了道的要求。道, 是天下之母, 是万物的缘由。《老子》 认为认识了道, 便可以从 “母体” 获知 “个体” 的内 涵、机制，抑或是原理，正如 “既得其母，以知其子” (第五十二章)。若想要长久的、很好的生存下去, 在 认识个体的时候, 也需要回溯母体 “道”, 遵循道的方 式也就是 “复守其母”, 就可以 “没身不殆”。“子”与 “母”, 是个体与整体的关系, 也是表面与内在的关系。 《老子》第三十三章说: “知人者智, 自知者明”, 能 了解他人虽是有智慧的, 但了解自己才能算得上明白 人。也就是说, 认识自己比了解他们更需要智慧, 认 识自己也是《老子》管理认识论中推己及人的前提和 基础。 


\section{5. 结语}

《老子》管理哲学是以探源的方式将管理现象的 分析提升到了形而上的层面。批判实在论代表学者巴 斯卡认为, 本体由三个层面构成, 分别是 “经验域、 实际域和真实域” ${ }^{[7]}$ 。经验域是人类感知对象的所在 层面, 是人类认识事物最初的接触, 位于本体的最上 面一层。真实域是整个结构中最深的一层。实际域在 经验域的下面一层, 是事物现象所在的层面, 也是所 有事件发生的层面。在真实域中, 存在着最为本源的 元素, 如机制、结构、力量和趋势。《老子》对管理的 认识路径, 是从实际域的管理现象入手去认识真实域 的管理机制、管理结构等。此过程中, 《老子》采取的 策略是将管理现象与自然现象相比拟, 将管理规律与 自然规律相对接。它在对管理观念、思想和逻辑的描 述上, 跨越了人为的定性与定量二分法, 将管理对象 放在人为设置的时间坐标之外, 将管理主客体同视为 自然之物、同受制于自然机制。

实证主义强调管理经验中的线性因果关系, 容易让人 产生感知的东西才是真实的狭险思维, 让科学结论局 限在具象之中, 其推演的管理理论的普适性也受到限 制。《老子》管理哲学的自然主义认识论, 另辟蹊径切 入管理世界的深层, 在一定程度上弥补了重视线性因 果关系的实证逻辑的不足, 为管理理论的变革和创新 提供了新的思路。

\section{REFERENCES}

[1] Wang J. (2012) Review of scientific epistemology in international basic education. Journal of Capital Normal University (NATURAL SCIENCE EDITION), 02: 34-39

[2] Xu C. (2011) The Tao of Laozi is the unity of ontology of existence and ontology of value. Philosophical research, 11: 55-59

[3] Tan L. (2018) The "multi-level unity" scientific view of critical realism. Philosophy of science and technology research, 04: 38-43

[4] Niu G. (2016) Explanation of Laozi. Commercial Press, Beijing.pp.34

[5] Song X. (2017) Interpretation of Laozi's "Xuantong" thought and the construction of social public body and life community. Chinese Taoism, 02: 50-53

[6] Li ruohui. (2016) Laozi's "action" theory. Philosophy trends, 08: 33-41

[7] Roy B. (2008) A Realist Theory of Science. Routledge, London and New York.pp.56 